\title{
Disruption of the peritrophic matrix by exogenous chitinase feeding reduces fecundity in Lutzomyia longipalpis females
}

\author{
Adriana Pereira Oliveira de Araújo, Erich Loza Telleria, Juliana da Matta Furniel Dutra, \\ Rute Maria Júlio, Yara Maria Traub-Csekö/+
}

Laboratório de Biologia Molecular de Parasitos e Vetores, Instituto Oswaldo Cruz-Fiocruz, Av. Brasil 4365, 21040-900 Rio de Janeiro, RJ, Brasil

Lutzomyia longipalpis is the most important vector of visceral leishmaniasis in Brazil. When female sandflies feed on blood, a peritrophic matrix (PM) is formed around the blood bolus. The PM is secreted by midgut cells and composed of proteins, glycoproteins and chitin microfibrils. The PM functions as both a physical barrier against pathogens present in the food bolus and blood meal digestion regulator. Previous studies of mosquitoes and sandflies have shown that the absence of a PM, resulting from adding an exogenous chitinase to the blood meal, accelerates digestion. In the present study, we analysed biological factors associated with the presence of a PM in L. longipalpis females. Insects fed blood containing chitinase (BCC) accelerated egg-laying relative to a control group fed blood without chitinase. However, in the BCC-fed insects, the number of females that died without laying eggs was higher and the number of eggs laid per female was lower. The eggs in both groups were viable and generated adults. Based on these data, we suggest that the absence of a PM accelerates nutrient acquisition, which results in premature egg production and oviposition; however, the absence of a PM reduces the total number of eggs laid per female. Reduced fecundity in the absence of a $P M$ may be due to inefficient nutrient conversion or the loss of the protective role of the PM.

Key words: Lutzomyia longipalpis - peritrophic matrix - chitinase

Phlebotomine insects are vectors of viral (Tesh 1988), bacterial and parasitic diseases (Adler \& Theodor 1957), including sandfly fever, bartonellosis and leishmaniasis. In Brazil, Lutzomyia longipalpis is the main vector of Leishmania infantum chagasi (Lainson \& Rangel 2005), which causes visceral leishmaniasis.

Previous molecular studies contributed to understanding the events that occur during the establishment of Leishmania infection in sandflies (Ramalho-Ortigao et al. 2010). Leishmania molecules such as LPG (Svarovska et al. 2010), which binds to the sandfly midgut galectin receptor PpGalec (Kamhawi et al. 2004), sandfly digestive enzymes (Sant'Anna et al. 2009, Telleria et al. 2010) and the peritrophic matrix (PM) (Pimenta et al. 1997) contribute to the success of the infection.

In the majority of sandfly species, the PM is formed between 12-24 $\mathrm{h}$ after blood ingestion (ABI) and degraded between 36-72 h ABI, when digestion is completed (Walters et al. 1995, Secundino et al. 2005, Sadlova \& Volf 2009). In L. longipalpis, PM formation begins $1 \mathrm{~h}$ ABI and is completed by $24 \mathrm{~h} \mathrm{ABI}$ (Secundino et al. 2005). The PM aides in the initial survival of Leishmania in the insect

Financial support: CNPq, CAPES, FAPERJ, FIOCRUZ

+ Corresponding author: ytraub@ioc.fiocruz.br

ELT current address: Yale School of Public Health,

New Haven, CT, USA

JMFD current address: Laboratório de Paleoparasitologia,

ENSP-Fiocruz, Rio de Janeiro, RJ, Brasil

Received 26 October 2011

Accepted 26 March 2012 by creating a proteolytic gradient between the midgut lumen and core of the food bolus (Pimenta et al. 1997).

PM degradation after blood digestion requires the activity of chitinases, which cleave the chitin microfibril components of the matrix. Midgut-specific chitinases (Llchitl and Ppchit1) from L. longipalpis and Phlebotomus papatasi were transcribed 72 h ABI (RamalhoOrtigao \& Traub-Cseko 2003, Ramalho-Ortigao et al. 2005). The maximum chitinolytic activity was detected in both species at approximately $48 \mathrm{~h}$ ABI (RamalhoOrtigao et al. 2005).

Previous studies on Aedes aegypti and Anopheles stephensi demonstrated that feeding insects with blood containing chitinase (BCC) or antibodies raised against PM proteins led to accelerated blood digestion, but did not interfere significantly with egg laying kinetics or the total number of eggs laid (Villalon et al. 2003). We investigated the effects of feeding exogenous chitinase on various biological factors of $L$. longipalpis.

Insects were collected at Gruta da Lapinha, Minas Gerais, Brazil, and maintained in our insectary (Brazil $\&$ Brazil 2003). Only the first offspring of the collected flies were used in the experiments. All animal procedures were approved by the Animal Care and Use Committee of Fiocruz (protocol P0-116-02). Insects were artificially fed through chick skin membranes placed on glass feeders. Control adult females were artificially fed hamster blood, while the experimental flies were fed hamster blood containing $1 \mu \mathrm{g} / \mathrm{mL}$ chitinase (Sigma Aldrich) (Villalon et al. 2003). Ten percent of the females were dissected $24 \mathrm{~h}$ ABI to investigate PM formation. PM presence was detected by optical microscopy; disruption of the PM was inferred when a PM was not observed by optical microscopy and the blood contents of the midgut dispersed read- 
ily throughout the dissecting buffer. An intact PM was not observed in BCC-fed flies. After the experimental feeding, females were separated into small containers and data were recorded daily until the death of all females. The data collected from three independent experiments included the egg laying kinetics, number of eggs laid and day of female death. Experiments 1, 2 and 3 had 27, 25 and 25 females in the control group and 35, 25 and 19 females in the experimental group, respectively. All insects were kept under the same humidity, temperature and photoperiod conditions. After all females died, the carcasses were removed from their containers. The egg hatching day and larval to adult development were also observed.

Statistical tests were conducted using GraphPad Prism version 5.01 (GraphPad Software, Inc). Survival analyses were performed using a chi-square test; all other analyses used a $t$ test to test for differences. Differences were considered statistically significant at $\mathrm{p}<0.05$.

Insect survival was not significantly affected by the disruption of the PM, although there was an increased survival trend for females fed BCC over 15 days (Fig. 1).

The disruption of the PM had an effect on the onset of oviposition. The average onset of oviposition occurred approximately two days earlier in chitinase-fed insects, which was significant different compared with the control group ( $t$ test, $\mathrm{p}<0.0001)$ (Fig. 2A). The number of females that laid eggs (at least 1 egg per female) was also investigated (Fig. 2B). Almost $60 \%$ of the chitinase-fed females died without laying eggs, while only $10 \%$ of the sandflies from the control group died without laying eggs $(\mathrm{p}=0.0107)$ (Fig. 2B).

The number of eggs laid per female was also investigated in relation to PM disruption. The mean number of eggs laid per BCC-fed female was approximately fivefold fewer than the control group (Fig. 3A) $(p<0.0001)$. Furthermore, fewer than $30 \%$ of the BCC-fed females laid eggs, while more than $50 \%$ of controls laid eggs (Fig. 3B) $(\mathrm{p}=0.0302)$.

Considering that $L$. longipalpis BCC-fed females started laying eggs earlier than the control group, but the number of barren females increased, we suggest that the accelerated digestion of the blood meal in the absence of a PM accelerates nutrient absorption, but prevents the full conversion of nutrients necessary for egg production. The role of the PM in digestion and its influence on nutrient acquisition has been studied in many insects. A complete digestive process requires the PM to compartmentalise digestive enzymes and midgut fluid fluxes. These fluxes are responsible for the translocation of enzymes and products of digestion (Terra 1988). However, for both A. aegypti and A. stephensi, early egg oviposition has not been observed in insects that ingested blood containing anti-PM sera (Villalon et al. 2003). Amino acid signalling through the nutrient-sensitive target of rapamycin (TOR) pathway has been shown to be essential for the activation of yolk protein precursor genes expression (Hansen et al. 2004), which is critical for egg development in A. aegypti. Thus, the disruption of the $\mathrm{PM}$ in BCC-fed sandflies may have influenced amino acid absorption, thereby altering the TOR signalling pathway and negatively impacting egg production.

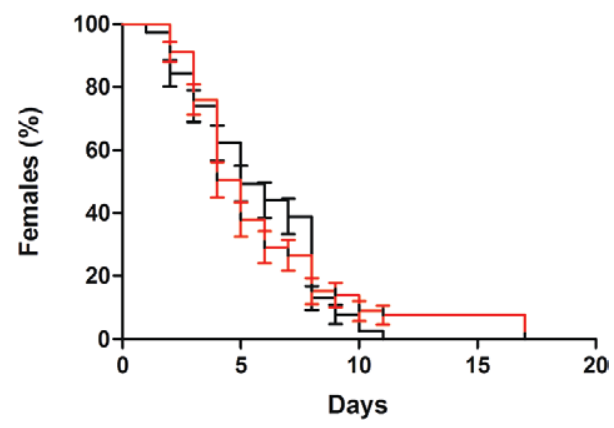

Fig. 1: effect of chitinase feeding on insect survival. Insect survival was recorded daily after blood ingestion. Mean values of three independent experiments are represented in the graphic. The black line indicates control group fed on blood only. The red line indicates experimental group fed on blood containing chitinase. Error bars are standard error of the mean. Flies lived beyond 12 days in only two of three experiments; therefore, error bars are not included on time points after 12 days.

A

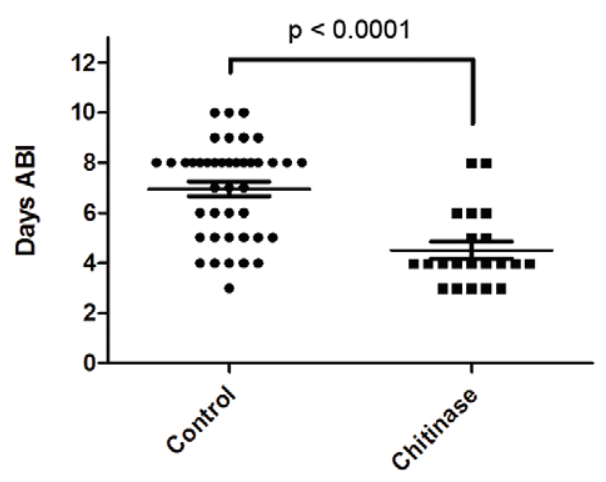

B

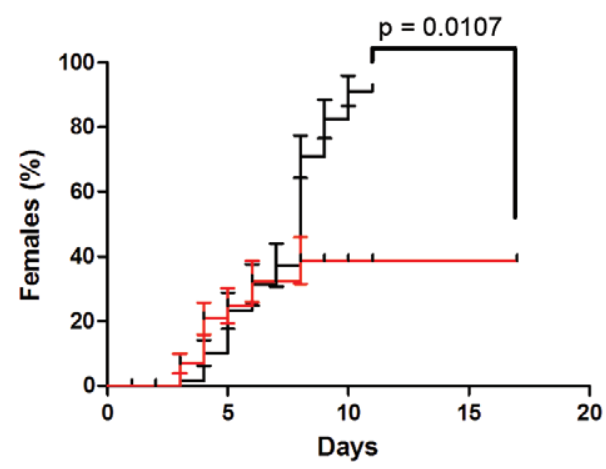

Fig. 2: effect of chitinase feeding on the onset of oviposition. The onset of egg laying was followed after blood ingestion (ABI). A: onset of egg laying for each female; B: percent of control vs. experimental flies laying eggs ABI. The black line indicates control group fed on blood only ( $\mathrm{n}$ $=42$ ). The red line indicates experimental group fed on blood containing chitinase $(n=21)$. Error bars are standard error of the mean.

Despite the earlier egg laying observed in the chitinase-fed females, a reduction in the total number of egg laying females and number of eggs laid per female was observed. Alterations in early blood digestion in the absence of a PM may have additional effects on the female 
A

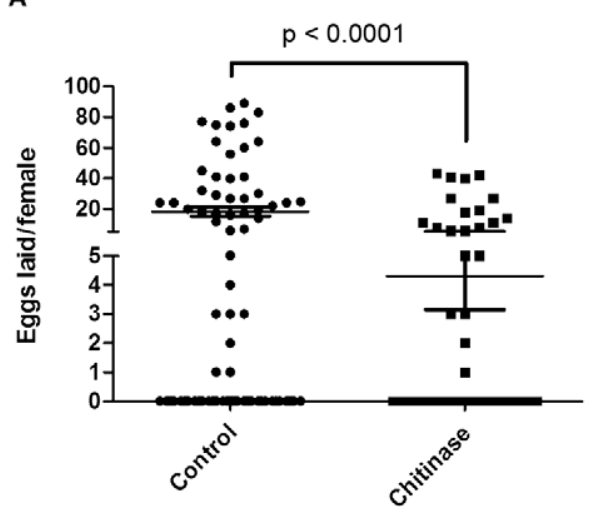

B

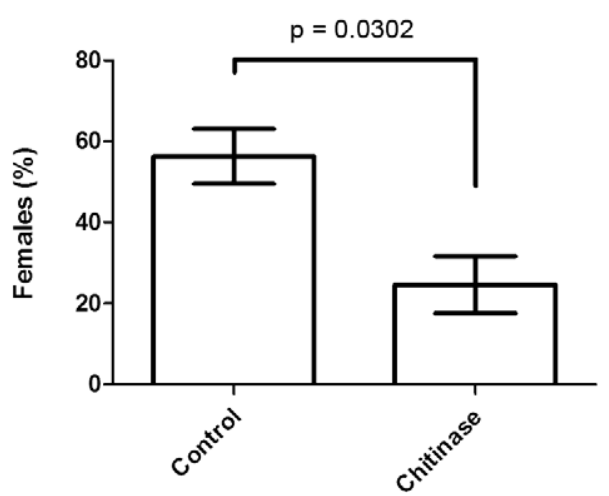

Fig. 3: effect of chitinase feeding on number of eggs laid. The number of eggs laid was counted after females died. A: number of eggs laid by each female; B: number of eggs laid by control vs. experimental populations. Error bars are standard error of the mean.

reproductive cycle. The direct contact of the gut epithelium with intermediates of haemoglobin digestion could induce oxidative stress in sandfly females, which was observed previously in Lutzomyia spinicrassa (Walters et al. 1995) and A. aegypti (Pascoa et al. 2002, Devenport et al. 2006) leading to compromised ability to produce eggs. In addition, the premature direct contact of the L. longipalpis gut microbiota with the midgut epithelium may cause an additional challenge to the BCC-fed insects because potentially pathogenic bacteria, such as Serratia marcescens, are present in the L. longipalpis midgut (Gouveia et al. 2008).

Our data contribute to understanding the role of the PM on insect biology by providing information for future midgut-targeted strategies to control sandflies.

\section{REFERENCES}

Adler S, Theodor O 1957. Transmission of disease agents by phlebotomine sandflies. Annu Rev Entomol 2: 203-226.

Brazil RP, Brazil BG 2003. Biologia de flebotomíneos neotropicais. In EF Rangel, R Lainson, Flebotomíneos do Brasil, Ed. Fiocruz, Rio de Janeiro, p. 257-274.

Devenport M, Alvarenga PH, Shao L, Fujioka H, Bianconi ML, Oliveira PL, Jacobs-Lorena M 2006. Identification of the Aedes aegypti peritrophic matrix protein AeIMUCI as a heme-binding protein. Biochemistry 45: 9540-9549.
Gouveia C, Asensi MD, Zahner V, Rangel EF, Oliveira SM 2008. Study on the bacterial midgut microbiota associated to different Brazilian populations of Lutzomyia longipalpis (Lutz \& Neiva) (Diptera: Psychodidae). Neotrop Entomol 37: 597-601.

Hansen IA, Attardo GM, Park JH, Peng Q, Raikhel AS 2004. Target of rapamycin-mediated amino acid signaling in mosquito anautogeny. Proc Natl Acad Sci USA 101: 10626-10631.

Kamhawi S, Ramalho-Ortigao M, Pham VM, Kumar S, Lawyer PG, Turco SJ, Barillas-Mury C, Sacks DL, Valenzuela JG 2004. A role for insect galectins in parasite survival. Cell 119: 329-341.

Lainson R, Rangel EF 2005. Lutzomyia longipalpis and the eco-epidemiology of American visceral leishmaniasis, with particular reference to Brazil - A Review. Mem Inst Oswaldo Cruz 100: 811-827.

Pascoa V, Oliveira PL, Dansa-Petretski M, Silva JR, Alvarenga PH, Jacobs-Lorena M, Lemos FJ 2002. Aedes aegypti peritrophic matrix and its interaction with heme during blood digestion. Insect Biochem Mol Biol 32: 517-523.

Pimenta PF, Modi GB, Pereira ST, Shahabuddin M, Sacks DL 1997. A novel role for the peritrophic matrix in protecting Leishmania from the hydrolytic activities of the sand fly midgut. Parasitology 115: 359-369.

Ramalho-Ortigao JM, Kamhawi S, Joshi MB, Reynoso D, Lawyer PG, Dwyer DM, Sacks DL, Valenzuela JG 2005. Characterization of a blood activated chitinolytic system in the midgut of the sand fly vectors Lutzomyia longipalpis and Phlebotomus papatasi. Insect Mol Biol 14: 703-712.

Ramalho-Ortigao JM, Saraiva EM, Traub-Cseko Y 2010. Sand fly - Leishmania interactions: long relationships are not necessarily easy. Open Parasitol J 4: 195-204.

Ramalho-Ortigao JM, Traub-Cseko YM 2003. Molecular characterization of Llchit1, a midgut chitinase cDNA from the leishmaniasis vector Lutzomyia longipalpis. Insect Biochem Mol Biol 33: 279-287.

Sadlova J, Volf P 2009. Peritrophic matrix of Phlebotomus duboscqi and its kinetics during Leishmania major development. Cell Tissue Res 337: 313-325.

Sant'Anna MR, Diaz-Albiter H, Mubaraki M, Dillon RJ, Bates PA 2009. Inhibition of trypsin expression in Lutzomyia longipalpis using RNAi enhances the survival of Leishmania. Parasit Vectors 2: 62.

Secundino NF, Eger-Mangrich I, Braga EM, Santoro MM, Pimenta PF 2005. Lutzomyia longipalpis peritrophic matrix: formation, structure and chemical composition. J Med Entomol 42: 928-938.

Svarovska A, Ant TH, Seblova V, Jecna L, Beverley SM, Volf P 2010. Leishmania major glycosylation mutants require phosphoglycans (lpg2-), but not lipophosphoglycan (lpg1-) for survival in permissive sand fly vectors. PLoS Negl Trop Dis 4: e580.

Telleria EL, de Araujo AP, Secundino NF, d'Avila-Levy CM, TraubCseko YM 2010. Trypsin-like serine proteases in Lutzomyia longipalpis - expression, activity and possible modulation by Leishmania infantum chagasi. PLoS ONE 5: e10697.

Terra WR 1988. Physiology and biochemistry of insect digestion: an evolutionary perspective. Braz J Med Biol Res 21: 675-734.

Tesh RB 1988. The genus Phlebovirus and its vectors. Annu Rev Entomol 33: 169-181.

Villalon JM, Ghosh A, Jacobs-Lorena M 2003. The peritrophic matrix limits the rate of digestion in adult Anopheles stephensi and Aedes aegypti mosquitoes. J Insect Physiol 49: 891-895.

Walters LL, Irons KP, Guzman H, Tesh RB 1995. Peritrophic envelopes of Lutzomyia spinicrassa (Diptera: Psychodidae). J Med Entomol 32: 711-725. 